\title{
UNIQUENESS OF HARMONIC MAPPINGS WITH BLASCHKE DILATATIONS
}

\author{
D. BSHOUTY, A. LYZZAIK AND A. WEITSMAN
}

\begin{abstract}
Let $\Omega$ be a bounded convex domain and let $\omega$ be a finite Blaschke product of order $N=1,2, \cdots$. It is known that the elliptic differential equation $\overline{f_{\bar{z}}} / f_{z}=\omega$ admits a one-to-one solution normalized by $f(0)=0, f_{z}(0)>0$ and maps the open unit disc $\mathbb{D}$ onto a convex $(n+2)$-gon whose vertices belong to $\partial \Omega$. In this paper it is shown that this solution is unique.
\end{abstract}

\section{INTRODUCTION}

A sense-preserving harmonic mapping $f$ of the open unit disk $\mathbb{D}$ is a solution of the elliptic differential equation

$$
\frac{\overline{f_{\bar{z}}(z)}}{f_{z}(z)}=\omega(z)
$$

where $\omega$, known as the analytic dilatation of $f$, is an analytic function in $\mathbb{D}$ with $\omega(\mathbb{D}) \subset \mathbb{D}$. A useful form of $f$ is

$$
f=h+\bar{g},
$$

where $h$ and $g$ are analytic functions in $\mathbb{D}$. In this case $\omega=g^{\prime} / h^{\prime}$ and the Jacobian of $f$ is

$$
J(f)=\left|h^{\prime}\right|^{2}-\left|g^{\prime}\right|^{2}=\left|h^{\prime}\right|^{2}\left(1-|\omega|^{2}\right) .
$$

It is known that $f$ is an open map, and that it is locally one-to-one except possibly at isolated points where it behaves locally like analytic functions near zero derivatives. We call $f$ univalent or locally univalent if it is one-to-one or locally one-to-one in $\mathbb{D}$ respectively.

The Riemann mapping theorem (RMT) states: If $|\omega|<k<1$ in $\mathbb{D}, \Omega$ is a bounded simply connected domain with a locally connected boundary, and if $w_{0} \in \Omega$, then there exists a univalent solution $f$ of (1)

1991 Mathematics Subject Classification. Primary 26C10, Secondary 30C15.

Key words and phrases. univalent harmonic mappings, Riemann mapping theorem.

The first author thanks the Promotion of Research Fund at the Technion for its support. 
that maps $\mathbb{D}$ onto $\Omega$ with $f(0)=w_{0}$ and $f_{z}(0)>0$. Moreover, if $\Omega$ is a Jordan domain, then $f$ extends to a homeomorphism from $\overline{\mathbb{D}}$ onto $\bar{\Omega}$.

If $\|\omega(z)\|_{\infty}=1$, then the RMT is no longer true and the following more general Riemann mapping theorem (GRMT) of Hengartner and Schober [6] holds.

Theorem A. Let $\Omega$ be a bounded simply connected domain with locally connected boundary, and let $w_{0}$ be a fixed point of $\Omega$. Also, let $\omega$ be an analytic function of $\mathbb{D}$ with $\omega(\mathbb{D}) \subset \mathbb{D}$. Then there exists a univalent harmonic mapping $f$ of $\mathbb{D}$ that satisfies the following properties:

(a) $f$ is a solution of (1).

(b) $f$ maps $\mathbb{D}$ into $\Omega$ with $f(0)=w_{0}$ and $f_{z}(0)>0$.

(c) The unrestricted limit $f^{*}\left(e^{i t}\right)=\lim _{z \rightarrow e^{i t}} f(z)$ exists and belongs to $\partial \Omega$ for all but a countable subset $E$ of the unit circle $\mathbb{T}=\partial \mathbb{D}$.

(d) The one-sided limits $\lim _{s \rightarrow t^{+}} f^{*}\left(e^{i s}\right)$ and $\lim _{s \rightarrow t^{-}} f^{*}\left(e^{i s}\right)$, through values $e^{i s} \notin E$, exist, belong to $\partial \Omega$, and are equal if $e^{i t} \notin E$ and distinct otherwise.

(e) The cluster set of $f$ at $e^{i t} \in E$ is the straight line segment joining the one-sided limits $\lim _{s \rightarrow t^{+}} f^{*}\left(e^{i s}\right)$ and $\lim _{s \rightarrow t^{-}} f^{*}\left(e^{i s}\right)$.

We remark that if $\|\omega\|_{\infty}<1$, then the GRMT reduces to the RMT.

For distinct points $c, d \in \mathbb{C}$, let $[c, d]$ denote the directed straight line segment from $c$ to $d$. For distinct points $c_{1}, c_{2}, \cdots, c_{N+2} \in \mathbb{C}$, where $N=1,2, \cdots$, the directed closed polygonal curve $\left[c_{1}, c_{2}, \cdots, c_{N+2}, c_{1}\right]=$ $\sum_{j=1}^{N+2}\left[c_{j}, c_{j+1}\right]$, with $c_{N+3}=c_{1}$, is called an $(N+2)-g o n$ whose vertices are the points $c_{j}$ and sides the segments $\left[c_{j}, c_{j+1}\right]$; here it is assumed that vertices and segments might be collinear. Also, we call $\left[c_{1}, c_{2}, \cdots, c_{N+2}, c_{1}\right]$ a Jordan or convex $(N+2)$-gon if it bounds a Jordan or convex region in $\mathbb{C}$.

In the case where $\Omega$ is convex and $\omega$ is a finite Blaschke product of order $N=1,2, \cdots$ of form

$$
\omega(z)=\lambda \prod_{k=1}^{N} \frac{z-p_{k}}{1-\bar{p}_{k} z} ; \quad\left|p_{k}\right|<1,|\lambda|=1,
$$

then results of W. Hengartner and G. Schober [7] and T. Sheil-Small [10] lead to the following theorem.

Theorem B. Let $\Omega$ be a bounded convex domain, and let $\omega$ be a Blaschke product of order $N=1,2, \cdots$. If $f$ is a function defined as in Theorem $A$, then $f(\mathbb{D})$ is the inner domain of a positively-directed convex $(N+2)$-gon $\left[c_{1}, c_{2}, \cdots, c_{N+2}, c_{1}\right]$ whose vertices $c_{j}$ lie on $\partial \Omega$, and the boundary function $f^{*}$ of $f$ is a step function defined by

$$
f^{*}\left(e^{i t}\right)=c_{j}, \quad t_{j-1}<t<t_{j}, \quad 1 \leq j \leq N+2,
$$


where

$$
0 \leq t_{0}<t_{1}<t_{2}<\cdots<t_{N+2}=t_{0}+2 \pi .
$$

Surprisingly, little is known about the uniqueness of the harmonic mapping $f$ described in the RMT and obviously in the GMRT. However, the uniqueness of $f$ in the RMT is known for each of the following cases:

(i) $\Omega$ is symmetric about the real axis, $\partial \Omega$ is sufficiently smooth, and the Maclaurin series of $\omega$ has real coefficients; see D. Bshouty, N. Hengartner and O. Hossian [4].

(ii) $\Omega$ is a strictly starlike domain; see D. Bshouty, N. Hengartner and W. Hengartner [3] and P. Duren [5, pp. 133-135] for a proof attributed to R. Kühnau.

(iii) $f_{z}(0)$ is fixed and $\partial \Omega$ is sufficiently smooth; see J. Gergen and F. Dressel [8], [9] and B. Bojarskii [1].

On the other hand, the uniqueness of $f$ in the GMRT is known only in the case where $\omega(z)=z^{n}$, where $n=1,2, \cdots$; see A. Weitsman [11].

The purpose of this paper is to extend the latter uniqueness result of [11] for the case where the dilatation $\omega$ is a finite Blaschke product. Our result states as follows.

Theorem 1. Let

(a) $\Omega$ be a bounded convex domain,

(b) $w_{0}$ be a fixed point of $\Omega$,

(c) $\omega$ be a finite Blaschke product of order $N=1,2, \cdots$, and

(d) $f$ be a function defined as in Theorem $A$.

Then $f$ is unique.

\section{Proof of Theorem 1.}

We establish the proof by contradiction. We assume without loss of generality that $w_{0}=0$. Suppose that there exist two different functions $f$ and $F$ defined as in Theorem A with $f_{z}(0) \leq F_{z}(0)$. Then, by Theorem $\mathrm{B}, f(\mathbb{D})$ and $F(\mathbb{D})$ are the inner domains of positively-directed convex $(N+2)$-gons $\left[c_{1}, c_{2}, \cdots, c_{N+2}, c_{1}\right]$ and $\left[d_{1}, d_{2}, \cdots, d_{N+2}, d_{1}\right]$ respectively, whose vertices $c_{j}$ and $d_{j}$ lie on $\partial \Omega$, and the boundary functions $f^{*}$ and $F^{*}$ are step functions defined by

$$
f^{*}\left(e^{i t}\right)=c_{j}, \quad t_{j-1}<t<t_{j}, \quad 1 \leq j \leq N+2,
$$

where

$$
0 \leq t_{0}<t_{1}<t_{2}<\cdots<t_{N+2}=t_{0}+2 \pi
$$

and

$$
F^{*}\left(e^{i s}\right)=d_{j}, \quad s_{j-1}<s<s_{j}, \quad 1 \leq j \leq N+2,
$$


where

$$
0 \leq s_{0}<s_{1}<s_{2}<\cdots<s_{N+2}=s_{0}+2 \pi
$$

Set

$$
\left\{\varphi_{j}: 0 \leq j \leq K+1\right\}=\left\{t_{j}: 0 \leq j \leq N+1\right\} \cup\left\{s_{j}: 0 \leq j \leq N+1\right\},
$$

where

$$
0 \leq \varphi_{0}<\varphi_{1}<\varphi_{2}<\cdots<\varphi_{K+2}=\varphi_{0}+2 \pi .
$$

It is immediate that $f^{*}\left(e^{i \varphi}\right)$ and $F^{*}\left(e^{i \varphi}\right)$ are constants on every interval $\left(\varphi_{j-1}, \varphi_{j}\right), 1 \leq j \leq K+2$. For each $0 \leq \lambda \leq 1$, let $P^{\lambda}=f-\lambda F$. Then $P^{\lambda}$ is a bounded harmonic mapping, $P^{\lambda}(0)=0$, and the boundary function $\left(P^{\lambda}\right)^{*}\left(e^{i \varphi}\right)=f^{*}\left(e^{i \varphi}\right)-\lambda F^{*}\left(e^{i \varphi}\right)$ of $P^{\lambda}$ is a step function on $\mathbb{T}$ that takes a constant value on every interval $\left(\varphi_{j-1}, \varphi_{j}\right), 1 \leq j \leq K+2$, Note that $\left(P^{\lambda}\right)^{*}$ attains at least two different step values, and that all the step values are nonzero in case $0 \leq \lambda<1$ since every $\lambda d_{j} \in \Omega$, $1 \leq j \leq N+2$. Thus every $P^{\lambda}, 0 \leq \lambda<1$, is a nonconstant function in $\mathbb{D}$. The cluster sets at the jumps of $P^{\lambda}$ are the line segments which we denote by $P^{\lambda}(\partial \mathbb{D})$.

We show that neither $P_{\bar{z}}^{\lambda}$ nor $P_{z}^{\lambda}$ can be identically zero in $\mathbb{D}$. If $P_{\bar{z}}^{\lambda}$ (or $P_{z}^{\lambda}$ ) is identically zero in $\mathbb{D}$, then $P^{\lambda}$ is analytic (or anti-analytic) in $\mathbb{D}$. Since $P^{\lambda}$ is bounded, $P^{\lambda}(0)=0$, and $\left(P^{\lambda}\right)^{*}$ is a step function, $P^{\lambda}$ is identically zero in $\mathbb{D}$ and we have a contradiction.

Thus $P_{\bar{z}}^{\lambda}$ is not identically zero in $\mathbb{D}$. But

$$
\overline{P_{\bar{z}}^{\lambda}}=\bar{f}_{\bar{z}}-\lambda \bar{F}_{\bar{z}}=\omega\left(f_{z}-\lambda F_{z}\right)=\omega P_{z}^{\lambda} .
$$

Hence, each $P^{\lambda}$ is an open sense-preserving harmonic mapping in $\mathbb{D}$ with analytic dilatation $\omega$. Since $|\omega|<1$, the argument principle applies (cf. [5, pp. 7-10]). In particular the notion of the order of a zero [5, p. 8] is well defined. Since $P^{\lambda}(0)=0$, it follows that whenever the origin does not belong to $P^{\lambda}(\partial \mathbb{D})$, the winding number for the positively oriented image satisfies

$$
n\left(P^{\lambda}(\partial \mathbb{D}), 0\right) \geq 1 .
$$

Assume that $0 \leq \lambda<1$ unless otherwise is specified. With an abuse of notation we denote by $a_{j}^{\lambda}$ and $b_{j}^{\lambda}, 1 \leq j \leq K+2$, the values of $\left(P^{\lambda}\right)^{*}\left(e^{i \varphi}\right)$ on the intervals $\left(\varphi_{j-1}, \varphi_{j}\right)$ and $\left(\varphi_{j}, \varphi_{j+1}\right)$, with $\varphi_{K+3}=\varphi_{1}$. This abuse comes from the fact that the $a_{j}^{\lambda}$ 's and $b_{j}^{\lambda}$ 's change roles on adjacent intervals.

Then the cluster set of $P^{\lambda}$ at each $e^{i \varphi_{j}}, 1 \leq j \leq K+2$, is the directed line segment $\ell_{j}^{\lambda}=\left[a_{j}^{\lambda}, b_{j}^{\lambda}\right]$ which is a point in case $a_{j}^{\lambda}=b_{j}^{\lambda}$. Note that $a_{j}^{\lambda}$ and $b_{j}^{\lambda}$ never coincide with the origin. With $\Gamma^{\lambda}=P^{\lambda}(\partial \mathbb{D})=\sum_{j=1}^{K+2} \ell_{j}^{\lambda}$, 
if none of the segments $\ell_{j}^{\lambda}$ contains the origin, then the winding number of $\Gamma^{\lambda}$ about the origin satisfies

$$
n\left(\Gamma^{\lambda}, 0\right)=\frac{1}{2 \pi} \sum_{j=1}^{K+2} \operatorname{Arg}\left(b_{j}^{\lambda} / a_{j}^{\lambda}\right),
$$

where $\operatorname{Arg}(\cdot)$ denotes the principal argument.

We proceed in the proof by establishing the following lemma which shows that $\Gamma^{\lambda}$ passes through the origin only for finitely many values $0 \leq \lambda<1$.

Lemma. Fix $1 \leq j \leq K+2$. Then there exist at most two values of $\lambda$ for which $\ell_{j}^{\lambda}$ passes through the origin.

(a) If no such value $\lambda$ exists, then $\operatorname{Arg}\left(b_{j}^{\lambda} / a_{j}^{\lambda}\right)$ is a continuous function on $[0,1)$.

(b) If exactly one such value $\lambda$, say $\lambda_{j}$, exists, then one of the following cases holds:

(i) $\operatorname{Arg}\left(b_{j}^{\lambda} / a_{j}^{\lambda}\right)$ is a continuous function on $[0,1)$ and

$$
\lim _{\lambda \rightarrow \lambda_{j}} \operatorname{Arg}\left(b_{j}^{\lambda} / a_{j}^{\lambda}\right)=\pi .
$$

(ii) $\operatorname{Arg}\left(b_{j}^{\lambda} / a_{j}^{\lambda}\right)$ is a continuous function on $[0,1)$ except for a jump discontinuity at $\lambda_{j}$ such that

$$
\lim _{\lambda \rightarrow \lambda_{j}^{-}} \operatorname{Arg}\left(b_{j}^{\lambda} / a_{j}^{\lambda}\right)=\pi \text { and } \lim _{\lambda \rightarrow \lambda_{j}^{+}} \operatorname{Arg}\left(b_{j}^{\lambda} / a_{j}^{\lambda}\right)=-\pi .
$$

(c) If two such values of $\lambda$, say $\lambda_{j}$ and $\mu_{j}$ with $\lambda_{j}<\mu_{j}$, exist, then $\operatorname{Arg}\left(b_{j}^{\lambda} / a_{j}^{\lambda}\right)$ is a continuous function on $[0,1)$ except for jump discontinuities at $\lambda_{j}$ and $\mu_{j}$ such that

$$
\lim _{\lambda \rightarrow \lambda_{j}^{-}} \operatorname{Arg}\left(b_{j}^{\lambda} / a_{j}^{\lambda}\right)=\pi \text { and } \lim _{\lambda \rightarrow \lambda_{j}^{+}} \operatorname{Arg}\left(b_{j}^{\lambda} / a_{j}^{\lambda}\right)=-\pi .
$$

and

$$
\lim _{\lambda \rightarrow \mu_{j}^{-}} \operatorname{Arg}\left(b_{j}^{\lambda} / a_{j}^{\lambda}\right)=-\pi \quad \text { and } \lim _{\lambda \rightarrow \mu_{j}^{+}} \operatorname{Arg}\left(b_{j}^{\lambda} / a_{j}^{\lambda}\right)=\pi .
$$

Proof. There are three distinct types of $\varphi_{j}:$ (1) $\varphi_{j}=s_{k} \neq t_{i}$ for some $1 \leq k \leq N+2$ and all $1 \leq i \leq N+2$, (2) $\varphi_{j}=t_{i} \neq s_{k}$ for some $1 \leq i \leq N+2$ and all $1 \leq k \leq N+2$, and (3) $\varphi_{j}=t_{i}=s_{k}$ for some $1 \leq i \leq N+2$ and some $1 \leq k \leq N+2$. We prove the lemma for each of these cases separately as follows.

(1) $\varphi_{j}=s_{k} \neq t_{i}$ for some $1 \leq k \leq N+2$ and all $1 \leq i \leq N+2$. In this case $a_{j}^{\lambda}=c_{r}-\lambda d_{k}$ and $b_{j}^{\lambda}=c_{r}-\lambda d_{k+1}$, where $c_{r}=f\left(e^{i \varphi_{j}}\right)$ for some $1 \leq r \leq N+2$ and $d_{N+3}=d_{1}$. By the convexity of $\Omega$, the points $\lambda d_{k}$ 
and $\lambda d_{k+1}$ lie in the open half-plane containing $\Omega$ and determined by a support line of $\Omega$ passing through $c_{r}$. Hence $\operatorname{Arg}\left(b_{j}^{\lambda} / a_{j}^{\lambda}\right)$ is a continuous function of $\lambda$ on $[0,1)$ and $\left|\operatorname{Arg}\left(b_{j}^{\lambda} / a_{j}^{\lambda}\right)\right|<\pi$ for every $0 \leq \lambda<1$. This gives Lemma 2.a for Case 1.

(2) $\varphi_{j}=t_{i} \neq s_{k}$ for some $1 \leq i \leq N+2$ and all $1 \leq k \leq N+2$. In this case $a_{j}^{\lambda}=c_{i}-\lambda d_{r}$ and $b_{j}^{\lambda}=c_{i+1}-\lambda d_{r}$, where $d_{r}=F\left(e^{i \varphi_{j}}\right)$ for some $1 \leq r \leq N+2$. Here we consider two cases:

(i) $d_{r}$ belongs to or lies on the left-hand side of the directed straight line from $c_{i}$ to $c_{i+1} ; d_{r}$ is an interior point of $\left[c_{i}, c_{i+1}\right]$ only if $\left[c_{i}, c_{i+1}\right]$ is a subset of $\partial \Omega$. Then, by the convexity of $\Omega$, the points $\lambda d_{r}$ lie on the left-hand side of the line and Lemma 2.a follows as in Case 1.

(ii) $d_{r}$ lies on the right-hand side of the directed straight line from $c_{i}$ to $c_{i+1}$. Then $\Im\left(c_{i+1} / d_{r}\right)>0$ and $\Im\left(c_{i} / d_{r}\right)<0$. Define the Möbius transformation

$$
T(\lambda)=\frac{b_{j}^{\lambda}}{a_{j}^{\lambda}}=\frac{c_{i+1}-\lambda d_{r}}{c_{i}-\lambda d_{r}} .
$$

A value $0 \leq \lambda<1$ for which $\ell_{j}^{\lambda}$ passes through the origin is precisely the same value for which $T(\lambda)$ attains a negative value. Since $T\left(c_{i+1} / d_{r}\right)=$ $0, T\left(c_{i} / d_{r}\right)=\infty, T$ maps the extended real line $\widehat{\mathbb{R}}$ to a positivelydirected circle $T(\widehat{\mathbb{R}})$ that separates the origin and infinity; it shall be assumed throughout that $T(\widehat{\mathbb{R}})$ is endowed with the direction inherited from the positive direction of $\widehat{\mathbb{R}}$ via $T$. Define the half-open subarc $C: w=T(\lambda), 0 \leq \lambda<1$, of $T(\widehat{\mathbb{R}})$. The statement of the lemma may be formulated to say that if $C$ intersects the negative real axis $\mathbb{R}^{-}=\{x: x<0\}$, then the first time is by moving from the upper half-plane to the lower half-plane. Since $T(0)=c_{i+1} / c_{i}, \Im T(0)>0$, $T(1)=\left(c_{i+1}-d_{r}\right) /\left(c_{i}-d_{r}\right)$, and $\Im T(1)<0$, the arc $C$ starts from the point $T(0)$ in the upper half-plane to the point $T(1)$ in the lower half-plane thus meeting $\mathbb{R}^{-}$exactly once in the desired manner. Hence Lemma 2.b.ii holds and the proof of Lemma 2 for Case 2 is complete.

(3) $\varphi_{j}=t_{i}=s_{k}$ for some $1 \leq i \leq N+2$ and some $1 \leq k \leq N+2$. In this case $a_{j}^{\lambda}=c_{i}-\lambda d_{k}$ and $b_{j}^{\lambda}=c_{i+1}-\lambda d_{k+1}$, where $c_{N+3}=c_{1}$ and $d_{N+3}=d_{1}$.

Suppose that $c_{i+1} d_{k}-c_{i} d_{k+1}=0$. Then $b_{j}^{\lambda} / a_{j}^{\lambda}=c_{i+1} / c_{i}=d_{k+1} / d_{k}$. Since $\Im\left(c_{i+1} / c_{i}\right)>0, \operatorname{Arg}\left(b_{j}^{\lambda} / a_{j}^{\lambda}\right)$ is a constant value in $(0, \pi)$ for every $0 \leq \lambda<1$ and Lemma 2.a holds.

For the rest of the proof we assume $c_{i+1} d_{k}-c_{i} d_{k+1} \neq 0$. Then

$$
T(\lambda)=\frac{b_{j}^{\lambda}}{a_{j}^{\lambda}}=\frac{c_{i+1}-\lambda d_{k+1}}{c_{i}-\lambda d_{k}}
$$


is a Möbius transformation which maps $\widehat{\mathbb{R}}$ homeomorphically onto an extended straight line or a circle. We argue as above in Case 2.ii. Define the subarc $C: w=T(\lambda), 0 \leq \lambda<1$, of $T(\widehat{\mathbb{R}})$ as above in 2.ii. Note that $T(0)=c_{i+1} / c_{i}, \Im T(0)>0, T\left(c_{i} / d_{k}\right)=\infty, T\left(c_{i+1} / d_{k+1}\right)=0$, and, by the convexity of $\Omega, c_{i} / d_{k}$ and $c_{i+1} / d_{k+1}$ do not belong to $[0,1)$. Hence, $C$ is a half-open directed straight line segment, a ray, or a directed circular arc that starts from $T(0)$ in the upper half-plane and avoids the origin. We consider three cases.

(a) $\Im\left(c_{i} / d_{k}\right) \Im\left(c_{i+1} / d_{k+1}\right)=0$. If $\Im\left(c_{i} / d_{k}\right)=0$, then, by the convexity of $\Omega, c_{i} / d_{k}<0$ and $C$ either is a directed straight line segment or a ray. In either case, $C$ either does not meet $\mathbb{R}^{-}$and Lemma 2.a holds, or meets it exactly once as desired and Lemma 2.b.ii holds.

If $\Im\left(c_{i+1} / d_{k+1}\right)=0$ and $\Im\left(c_{i} / d_{k}\right) \neq 0$, then, by the convexity of $\Omega$, $c_{i+1} / d_{k+1}<0$ and $T(\widehat{\mathbb{R}})$ is a circle that passes through the origin. Since $0 \notin C, C$ either does not meet $\mathbb{R}^{-}$and Lemma 2.a holds, or meets it once only if $T(\widehat{\mathbb{R}})$ is positively-directed.

(b) $\Im\left(c_{i} / d_{k}\right) \Im\left(c_{i+1} / d_{k+1}\right)>0$. In this case $T(\widehat{\mathbb{R}})$ is a circle that does not separate the origin and $\infty$. Then $C$ either does not meet $\mathbb{R}^{-}$and Lemma 2.a holds, meets it once and Lemma 2.b.i holds, or meets it twice and Lemma 2.c holds.

(c) $\Im\left(c_{i} / d_{k}\right) \Im\left(c_{i+1} / d_{k+1}\right)<0$. In this case $T(\widehat{\mathbb{R}})$ is a circle that separates the origin and $\infty$.

If $T(\widehat{\mathbb{R}})$ is positively-directed, then $C$ either does not meet $\mathbb{R}^{-}$and Lemma 2.a holds, or meets it once and Lemma 2.b.i holds.

If $T(\widehat{\mathbb{R}})$ is negatively-directed, then $0<\operatorname{Arg}\left(c_{i} / d_{k}\right)<\pi,-\pi<$ $\operatorname{Arg}\left(c_{i+1} / d_{k+1}\right)<0$, and, by the convexity of $\Omega, c_{i}$ and $c_{i+1}$ are interior points of the subarc of the positively-directed $\partial \Omega$ with initial and terminal points $d_{k}$ and $d_{k+1}$ respectively. Moreover, we see that $\operatorname{Arg}(T(\lambda))$ is decreasing in $\lambda$ and $\operatorname{Arg}(T(1)) \geq-\pi$ which implies that $C$ does not meet $\mathbb{R}^{-}$and Lemma 2 .a holds.

This proves Lemma 2 for Case 3, and the proof of Lemma 2 is complete.

Now we complete the proof of Theorem 1. It follows from the Lemma that $n\left(\Gamma^{\lambda}, 0\right) \leq n\left(\Gamma^{0}, 0\right)$ for $0<\lambda<1$. Indeed, by the Lemma, $n\left(\Gamma^{\lambda}, 0\right)$ varies continuously with $\lambda$ on intervals until perhaps some $\ell_{j}$ passes through the origin. Since $n\left(\Gamma^{\lambda}, 0\right)$ is integer valued, it must be constant on the intervals of continuity. For the exceptional values of $\lambda=\lambda_{j}$, then $n\left(\Gamma^{\lambda}, 0\right)$ undergoes a jump decrease in both cases ii) and iii), then a compensating jump increase in case iii). Since $n\left(\Gamma^{0}, 0\right)=1$, equation (2) yields $n\left(\Gamma^{\lambda}, 0\right)=1$ for all $0 \leq \lambda<1$. 
Suppose that $\lambda=f_{z}(0) / F_{z}(0)<1$. Then $P_{z}^{\lambda}(0)=0$ and, consequently, $P_{\bar{z}}^{\lambda}(0)=0$ since $P^{\lambda}$ is a sense-preserving harmonic mapping. Hence, the Jacobian of $P^{\lambda}$ at the origin is zero, $P^{\lambda}$ has a zero of order at least 2 at the origin, and $n\left(\Gamma^{\lambda}, 0\right) \geq 2$. This yields a contradiction and $f_{z}(0)=F_{z}(0)$.

Evidently, the function $P^{\lambda}$ converges uniformly in $\mathbb{D}$ to the function $P^{1}=f-F$ as $\lambda \rightarrow 1^{-}$. We are assuming, contrary to Theorem 1 that $P^{1} \not \equiv 0$ so that $P^{1}$ then also has dilatation $\omega$. By the argument used in the previous paragraph, $P^{1}$ has a zero of order $m \geq 2$ at the origin. Since $P^{1}$ is locally one-to-one outside a discrete set, there exists a sufficiently small circle $C$ centered at the origin on which $P^{1}$ is never zero and $n\left(P^{1}(C), 0\right)=m$. Then for $\lambda$ sufficiently close to 1 ,

$$
m=n\left(P^{1}(C), 0\right)=n\left(P^{\lambda}(C), 0\right) \leq n\left(\Gamma^{\lambda}, 0\right)=1,
$$

contradicting the fact that $m \geq 2$.

It must then be that $f \equiv F$, and the proof of Theorem 1 is complete.

\section{Concluding Remarks}

Regarding the general question of uniqueness in the GRMT, it is possible that the condition (c) of Theorem 1 might be relaxed to simply $\omega(\mathbb{D}) \subset \mathbb{D}$, but we are unable to do this. It seems more difficult to predict if the convexity condition (a) is really needed. An example of nonuniqueness in this case was claimed by the third author in the Bull. Lond. Math. Soc., 31 (1999), but it contains an error and the problem remains open.

\section{REFERENCES}

[1] B. V. Bojarskii, Generalized solutions of a system of differential equations of first order and elliptic type with discontinuous coefficients, Mat. Sb. (N.S.) 43 (85), 1957, 451-503.

[2] D. Bshouty and W. Hengartner, Boundary values versus dilatations of harmonic mappings, J.Analyse Math., 72, 1997, 141-164.

[3] D. Bshouty, N. Hengartner and W. Hengartner, A constructive method for starlike harmonic mappings, Numer. Math., 54, 1988, 167-178.

[4] D. Bshouty, W.Hengartner and O.Hossian, Harmonic typically real mappings, Math. Proc. Cambridge Philos. Soc., 119, 1996, 673-680.

[5] P. Duren, Harmonic mappings in the Plane, Cambridge University Press, 2004.

[6] W. Hengartner and G. Schober, Harmonic mappings with given dilatation, J. London Math. Soc., 33, 1986, 473-483.

[7] W. Hengartner and G. Schober, On the boundary behavior of orientationpreserving harmonic mappings, Complex Variables Theory Appl., 5, 1986, $197-208$. 
[8] J. J. Gergen and F. G. Dressel, Mapping by p-regular functions, Duke Math. J., 18, 1951, 185-210.

[9] J. J. Gergen and F. G. Dressel, Uniqueness for p-regular Mapping, Duke Math. J., 19, 1952, 435-444.

[10] T. Sheil-Small, On the Fourier coefficients of a step function, Mich. Math. J., 36 (1989), 459-475.

[11] A.Weitsman, On univalent harmonic mappings and minimal surfaces, Pacific J. Math., 192, 2000, 191-200.

Department of Mathematics, Technion, Haifa 32000, Israel

E-mail address: Daoud@tx.technion.ac.il

Department of Mathematics, American University of Beirut, Beirut, LEBANON

E-mail address: lyzzaik@aub.edu.lb

Department of Mathematics, Purdue University, West Lafayette, IN 47907-1395, USA

E-mail address: weits@math.purdue.edu 